\title{
La crisis de la normalidad en el contexto socioeconómico capitalista
}

\section{The crisis of normality in the capitalist socio-economic context}

\section{A crise de normalidade no contexto socioeconômico capitalista}

\author{
Angel V. Guajardo Romero \\ Universidad de Playa Ancha de Ciencias de la Educación \\ angelvioleta.quajardo@gmail.com \\ https://orcid.org/0000-0002-5213-2203
}

\begin{abstract}
RESUMEN
La inacción ha permitido una reflexión sin precedentes, en especial en los países periféricos, en donde las desigualdades propias del sistema socioeconómico, se han acelerado y exacerbado con la actual crisis sanitaria. El análisis crítico de la normalidad, permite la discusión sobre los valores, que han llevado a la reproducción de fenómenos nefastos para la vida. Para muchos autores, este es un momento en donde se abre la posibilidad política de disputar las tendencias futuras de desarrollo. La nueva normalidad, se presenta como una posibilidad impuesta desde arriba o como una construida desde las bases, en donde es clave la creación de un nuevo marco conceptual, coherente con la responsabilidad política actual, que les entregue a los movimientos sociales, el sustento teórico para su renovación y para la creación de nuevas estrategias, que permitan dar una respuesta democrática de base, ante las tendencias de represión y de violencia estatal.
\end{abstract}

\begin{abstract}
Inaction has allowed unprecedented reflection, especially in peripheral countries, where inequalities inherent to the socioeconomic system have accelerated and exacerbated with the current health crisis. The critical analysis of normality, allows the discussion about the values that have allowed the reproduction of phenomena harmful to life. For many authors, this is a time when the political possibility opens up, to dispute future development trends. The new normality is presented as a possibility imposed from above or as one built from the grassroots where the key is the creation of a new conceptual framework, consistent with the current political responsibility, which provides social movements, with the theoretical support for their renewal and for the creation of new strategies. that allow a basic democratic response to the tendencies of repression and state violence.
\end{abstract}

\section{RESUMO}

A inação tem permitido uma reflexão sem precedentes, principalmente nos países periféricos, onde as desigualdades inerentes ao sistema socioeconômico foram aceleradas e exacerbadas pela atual crise de saúde. A análise crítica da normalidade permite a discussão de valores, que têm levado à reprodução de fenômenos prejudiciais à vida. Para muitos autores, este é um momento em que se abre a possibilidade política de disputar as tendências futuras de desenvolvimento. A nova normalidade se apresenta como uma possibilidade imposta de cima ou construída a partir da base, onde a criação de um novo marco conceitual, consistente com a responsabilidade política atual, é fundamental para dar suporte teórico sociales, 3(5), 5-13. https://dx.doi.org/10.15381/espiral.v3i5.18682 
aos movimentos sociais. pela sua renovação e pela criação de novas estratégias que permitam dar uma resposta democrática de base às tendências de repressão e violência do Estado.

PALABRAS CLAVES: Normalidad; desigualdad; crisis sanitaria; reflexión crítica; sistema socioeconómico.

KEYWORDS: Normality; inequality; health crisis; critical reflection; socioeconomic system.

PALAVRAS-CHAVE: Normalidade; desigualdade; crise de saúde; reflexão crítica; sistema socioeconômico.

\section{Introducción}

El concepto de normalidad ha impregnado la discusión intelectual y social en el último tiempo, particularmente en el contexto chileno, la crítica a la normalidad se puede observar en las manifestaciones sociales a partir de octubre de 2019, no solo como una crítica profunda al sistema socioeconómico, sino que, también como una llamada de atención sobre la carga ideológica que puede tener este concepto.

Y lo que de una u otra forma ha podido plantear el despertar social en Chile, con la actual crisis sanitaria ha quedado en evidencia. Los efectos de la pandemia, sacan a la luz las infinitas interconexiones e interdependencias, producto de vivir en un mundo extremadamente globalizado, así como las profundas desigualdades propias del capitalismo.

El análisis crítico de la normalidad, plantea la necesidad de una reflexión acerca de: la relación establecida entre el mercado, la política, el Estado, la sociedad y el medio ambiente, el rol de las personas como sujetos de acción y cambio sociopolítico y de los valores que sustentan lo que se concibe como lo aceptable o deseable dentro de la sociedad.

Es así como surge la pregunta de investigación: ¿Es posible hablar de una crisis de la normalidad, en el contexto socioeconómico capitalista? Para dar respuesta a esta interrogante, en primer lugar, se presenta una revisión etimológica y semántica del concepto de normalidad, que no pretende ser exhaustiva, pero que permite entender como este concepto se fue transformando a través del tiempo y como llega a ser en la actualidad un concepto que permea toda la discusión sobre la realidad social. En segundo lugar, se plantea un análisis de las reflexiones intelectuales producidas a propósito de la actual crisis sociosanitaria, provenientes de distintas partes del mundo, pero especialmente desde América Latina, lo que ayuda a entender desde el punto de vista de su frecuencia y reproducción, los elementos que conforman lo que socialmente concebimos como normalidad. Posteriormente se revisan las posibles alternativas que plantea la nueva normalidad y el escenario pospandemia. Finalmente se presentan algunas reflexiones, sobre la responsabilidad política que conlleva este momento de profundas incertidumbres, pero también de apertura política para un cambio estructural de la sociedad.

\section{Metodología}

El presente trabajo surge del análisis crítico de la construcción social del concepto de normalidad en el contexto socioeconómico capitalista. Para responder a la pregunta de investigación planteada, sobre una posible crisis de la normalidad, entendida como la crisis de los valores que han normalizado fenómenos que pueden ir en contra del principio fundamental de la vida, se desarrolló en primer lugar, una revisión bibliográfica sobre el surgimiento de los conceptos de normal y normalidad y de sus usos dentro de las ciencias sociales. En segundo lugar, se realizó un análisis de contenido, de las reflexiones intelectuales acerca de la actual crisis socio sanitaria. Para lo anterior se utilizaron artículos, entrevistas, simposios y conversatorios, de diferentes países, pero en especial desarrollados en América Latina. 


\section{Construcción social del concepto de normalidad}

El concepto de normalidad presenta una serie de dificultades a la hora de intentar definirlo, lo que deriva de sus características propias y de los usos que se le ha dado tanto en el ámbito de las ciencias naturales, como sociales.

De acuerdo a la investigación de Hacking (1991), la primera aparición de la palabra en un diccionario define a lo normal como: lo usual, regular, común o típico y sus primeros usos se encuentran en medicina, donde lo normal nace como lo opuesto a lo patológico, pero se puede observar que su significado actual lo adquiere a partir de la década de 1820.

De acuerdo al principio de Broussais, lo patológico es definido como la desviación con respecto a lo normal. Es Comte tomando los planteamientos de Broussais, quién traslada el concepto al mundo social, posteriormente pasa a utilizarse en la mayoría de los ámbitos de la vida, Hacking (1991) considera que la palabra se hizo indispensable porque creaba cierta objetividad con respecto a los asuntos humanos.

Cuando el concepto de normalidad es trasladado al mundo político, se produce un cambio en su significado. Si en medicina lo normal es sinónimo de salud, en el mundo sociopolítico lo normal es el estado al cual se debía aspirar, el deber ser. Esta consideración crea una tensión en el concepto de normal: lo normal como promedio y lo normal como figura de perfección a la cual se debía aspirar, ya que dice como son las cosas, pero también como deberían ser (Hacking, 1991).

Durkheim al igual Comte considera que, el par normal - patológico se puede extender al análisis de los fenómenos poblacionales por medio de la mediación estadística. De esta forma, surge la asociación de tres conceptos claves para entender este planteamiento: frecuencia, normalidad y moralidad. Durkheim reconoce la existencia de las regulaciones matemáticas y una anterioridad lógica de lo patológico, de la cual deriva el carácter normativo de lo normal, pues viene de la conciencia, de la existencia de riesgos a ser prevenidos (Hacking, 1991).

Canguilhem (1971), considera que los conceptos de normal y patológico tienen una relación especular que llama de polaridad, ambos conceptos necesitan el uno del otro para afirmarse. La polaridad es considerada como una característica inherente de los conceptos que constituyen un valor. De esta forma si lo normal es lo preferible o deseable, lo anormal será considerado como lo detestable.

Para Durkheim (1986), es la frecuencia estadística la que se utiliza como parámetro de normalidad, de acuerdo a lo anterior, un fenómeno social será considerado normal si es frecuente y es precisamente esa frecuencia la que le concede un valor moral, pues se considera que es la expresión de las exigencias colectivas. De esta forma, la media muestra la regla y al mismo tiempo posibilita que se multiplique, pues se presenta como un modelo a seguir. En este sentido, es posible observar que la frecuencia estadística muestra las normas sociales y establece un vínculo entre el ser y el deber ser.

En tanto para otros autores lo normal no sería un concepto estático ni neutral, por lo tanto, no es posible considerar a la frecuencia estadística como un criterio de demarcación universal univoco. De acuerdo a lo planteado por Caponi (1998), para Canguilhem, no es la media estadística la que establece lo normal, porque a pesar de que puede servir para decir lo que ocurre en una determinada sociedad y momento histórico, no debe asumirse como un criterio de demarcación entre estados sociales considerados como patológico o normales. Por su parte, Hacking (1991) considera que el concepto de normal, a partir del siglo XX se habría convertido en un poderoso instrumento ideológico, pues lo normal indica lo que es típico, el promedio objetivo, pero también hace alusión a lo que debiera ser el destino escogido. 
En relación a estos últimos planteamientos, es posible observar cómo se va configurando la construcción social de este concepto en la actualidad y permite analizar cómo la proliferación de ciertas tendencias propias del sistema capitalista, son consideradas como normales desde el punto de vista de su frecuencia, pero nefastas desde un punto de vista moral.

\section{Elementos constitutivos de la normalidad}

Para poder entender la construcción social de lo normal y los elementos constitutivos de la normalidad que estarían entrando el crisis, se toman los planteamientos de varios autores (Dussel, 2020; Chomsky, 2020; Miley, 2020; Torres 2020; Tomadoni, 2020) que consideran que la humanidad está viviendo un momento histórico inédito, una crisis de civilización, una profunda crisis existencial, producto del desarrollo de un nuevo tipo de crisis macro estructural que se ha acentuado y acelerado con la expansión de la pandemia y que está determinada por la consecución y contraposición de la crisis del cambio climático, la crisis de representación del sistema político, la crisis de polarización geopolítica y todo lo anterior permeado por la crisis terminal del capitalismo.

Es la lógica neoliberal la que se pone en cuestión de manera radical, porque se considera que pone en peligro la vida en todo el planeta. Existe toda una filosofía que justifica el capitalismo financiero (Dussel, 2020), que responde por sobre todo al aumento de la tasa de ganancia del capital, pero esta lógica que limita la intervención del estado, no ha podido responder ante la irrupción y el avance de la pandemia. De acuerdo a esta idea, es el capital privado en el mercado, quien debe solucionar el problema sanitario, pero ningún sistema privado de salud puede hacerse cargo de una pandemia a nivel mundial, entonces necesariamente tienen que entrar los Estados a resolver y hacerse cargo de un sistema de salud público debilitado y en corporalidades deterioradas por una industria alimentaria, que obedece a este mismo razonamiento de aumento de ganancias y no de aumento cualitativo la vida.

\section{Desigualdades socioeconómicas}

Siguiendo a Cohen (2020), la desigualdad se presenta como un fenómeno aún más peligroso que la pobreza, ya que viene de las estructuras sociales y es la muestra de la falta de consenso y voluntad política para el resguardo de la vida. En este sentido, las desigualdades propias del capitalismo por desposesión, se pueden analizar como uno de los principales elementos constitutivos de nuestra normalidad, que se aceleran y exacerban con la actual crisis sanitaria.

Con respecto a las desigualdades socioeconómicas, podemos observar que es el sector informal de la economía, él que se ha visto más afectado por la crisis sanitaria, ya que para muchas personas el hecho de no trabajar y quedarse en casa, significa perder los medios de subsistencia. La situación preexistente indica que, existe una normalización en los países periféricos, en donde el sector informal represente un parte considerable de la economía, lo que significa que muchas personas no cuentan con las garantías básicas, para trabajar y subsistir.

\section{Desigualdad en infraestructura y en el acceso a servicios básicos}

Las principales desigualdades territoriales que se evidencias en este contexto, están relacionadas con la organización de las ciudades y el acceso al agua. Estos dos ámbitos han quedado en evidencia, debido a que las medidas sanitarias tomadas por los gobiernos, que demandan, por una parte, quedarse en casa, situación que es imposible para los millones de personas que no tienen un hogar, un distanciamiento físico que es difícil de respetar en ciudades con alta densidad poblacional, y por otra, la necesidad de medidas de higiene, que requieren de la disposición de agua. 
El problema de la desigualdad en infraestructura, está también relacionado con la desigualdad en salud, desigualdades que existen al interior de los países, pero también entre ellos. Al respecto, resulta interesante el aporte de Cohen (2020), para entender la necesidad de repensar la ciudad y de Meneses (2020) en relación a la desigualdad en salud que se observa en África. Cohen habla sobre la diferencia que hay entre las inversiones en infraestructura dentro de las ciudades, plantea que, por una parte, proliferan los edificios en altura y las grandes carreteras, pero por otra, no existen las suficientes camas en los hospitales, una necesidad de salud básica para enfrentar grandes crisis sanitarias. Esta falta de previsión y responsabilidad de algunos gobiernos, se agrava si se considera que existía una advertencia con respecto a la actual pandemia (Informe anual sobre preparación mundial para las emergencias sanitarias. Junta de Vigilancia Mundial de la Preparación, 2019). Con respecto, las desigualdades en salud al interior de los países, éstas derivan de la existencia de un débil sistema de salud pública, que no es capaz de asegurar la salud de las personas más vulnerables y otro privado que mejora las condiciones para quien puede pagar por ella. Las desigualdades entre países resultan aún más profundas, si se piensa en el acceso a una virtual vacuna o si se considera que en África hay otras pandemias como la tuberculosis, que matan más que el Covid-19 y para las cuales ya existe una vacuna (Meneses, 2020).

\section{Desigualdades de género}

La cadena de desigualdades que enfrentan las mujeres, también han quedado en evidencia en el actual contexto, de acuerdo a la investigación de Batthyány (2020), el impacto de la crisis sanitaria en el ámbito del género, se expresa principalmente en tres dimensiones: los cuidados, el mercado de trabajo y la violencia de género.

Es posible observar que, las labores de cuidados se presentan como uno de los ámbitos más débiles de la sociedad y que se ha intensificado tanto en los hogares como en el sistema sanitario. Para las mujeres esto implica una situación de vulnerabilidad frente al confinamiento, debido a la dificultad para conciliar sus tareas remuneradas, con las tareas domésticas, las de cuidados y las de seguimiento escolar. Siguiendo el análisis de Batthyány (2020), la crisis sanitaria muestra con mucha claridad, como el trabajo de los cuidados está subvencionando tanto servicios públicos del estado, como beneficios privados para el sector de las empresas, por medio del trabajo femenino no remunerado.

\section{Violencia hacia grupos vulnerables}

El aumento de la violencia de género es exponencial, violencia que preexistía pero que se ha agravado con la crisis sanitaria, debido a que las medidas de confinamiento obligan a las mujeres víctimas de violencia intrafamiliar, a permanecer por más tiempo del habitual con sus agresores. Además de la violencia de género Batthyány (2020), expone que la violencia hacia otros grupos vulnerables también ha aumentado, como la violencia hacia las disidencias sexuales, a las personas con discapacidad, en especial hacia las niñas y mujeres con discapacidad y la violencia hacia la población migrante, lo que también está relacionado con el aumento de las tensiones al interior de los hogares derivadas de las medidas de confinamiento.

\section{Violencia estatal}

La violencia como elemento constitutivo de la normalidad, también se expresa en otras esferas de la vida social, de acuerdo al estudio realizado por Miley (2020), sobre movimientos de resistencia, se muestra que ciertas tendencias de violencia estatal hacia estos grupos se han profundizado con la crisis sanitaria. En primer lugar, se observa una exacerbación de las tendencias violentas del estado hacia estas 
comunidades, usando como pretexto las medidas de confinamiento. En segundo lugar, la polarización política y el revanchismo racista contra los pueblos originarios ha empeorado en este periodo, el caso del movimiento indigenista en Bolivia y del pueblo Mapuche en Chile son dos claros ejemplos en la región.

\section{Polarización geopolítica}

Miley (2020) y Chomsky (2020), coinciden al considerar que uno de los peligros más graves que enfrenta la sociedad en la actualidad, es la polarización geopolítica, caracterizada por: la mantención de armamento de destrucción masiva por parte algunos países, así como por un estado de guerra permanente en países de oriente medio y África, que hasta la fecha han cobrado millones de muertes. Chomsky (2020) es radical al decir que, tanto la amenaza de la guerra nuclear, como el calentamiento global, son dos peligros que la humanidad viene enfrentando desde hace unas décadas y que son mucho más graves que el Covid-19, al ser eventos que podrían terminar con la civilización.

\section{¿Qué nos depara el futuro, la nueva normalidad como una realidad impuesta o como una realidad que construimos en comunidad?}

Mucho se ha habladoúltimamente de la nueva normalidad, para hacer referencia a las medidas y normas sociales que deberían regir en el escenario pospandemia. La nueva normalidad habla, por una parte, de las medidas de higiene que las personas deben incluir en sus rutinas diarias, del distanciamiento físico que se debe resguardar en el espacio público, del uso indiscriminado de mascarillas, de la apertura para ir al trabajo o al mercado, pero de serias restricciones para realizar actividades de esparcimiento. Pero, por otra parte, habla de fenómenos que pueden poner en un profundo riesgo la democracia y el estado de derecho, como: el reforzamiento de estados autoritarios protectores o agresores (Torres, 2020), ya sea de índole social o neoliberal, el establecimiento del estado de excepción permanente legitimado por un movimiento mundial de autoconservación, basado en el miedo a la muerte por contagio a este virus o a los que vendrán y a la imposición de tecnologías de vigilancia carcelarias como un tipo de panóptico mundial.

Se habla de la nueva normalidad, porque de una u otra forma, desde el imaginario colectivo se intuye que no es posible volver a la normalidad anterior, pero quizá la causa sea diferente a la pregonada por los medios de comunicación.

Si bien es cierto, se está entrando en un periodo de mucha incertidumbre y de posibilidades muy peligrosas, en términos de represión y vigilancia, en donde el poder estatal es capaz de controlar a las personas, reprimir comportamientos y de moldear subjetividades, también es un periodo de profundas modificaciones, de apertura del espacio político, que da la posibilidad de disputar las tendencias futuras de desarrollo y de intervenir en la agenda política (Domingues, 2020).

En este sentido, resulta crucial observar la resistencia desde los márgenes sociales (Miley, 2020), puesto que esas respuestas construidas desde las territorialidades, muestran una forma de enfrentar a un sistema que está agotado y que es insostenible para la reproducción de la vida. Estas respuestas también están relacionadas con: la revalorización de lo público y lo común, la reconstrucción de lazos comunitarios, basados en la solidaridad y en la búsqueda de igualdad y equidad (Batthyány, 2020), el humanismo, la autodeterminación de los pueblos, la sabiduría ancestral y las corrientes feministas, que platean formas de producción más solidarias y economías que sean capaces de resguardar tanto la vida como el medio ambiente, en definitiva una respuesta que ponga a la vida, como el eje central de la vida. 


\section{Recomendaciones}

De acuerdo al análisis realizado, es posible observar que actualmente vivimos en un momento de profundas crisis que pueden interactuar de forma inesperada. En este sentido, es posible considerar que estamos ante una crisis de la normalidad concebida desde un contexto socioeconómico capitalista.

Desde el punto de vista semántico, el concepto de normalidad es contradictorio y presenta una serie de dificultades en su definición, pues más que hacer referencia a una definición unívoca, hace referencia a una definición que es histórica y socialmente cambiante, por lo que lo normal y patológico no pueden asumirse como criterios de demarcación universal.

Desde el punto de vista de la construcción social del concepto y de los usos ideológicos que se le puede dar, la normalidad representa lo que es frecuente, pero además representa un objetivo a alcanzar, lo aceptable o deseable dentro de una sociedad. Lo anterior plantea una serie de cuestionamientos, si los individuos que conforman determinada sociedad, no presentan una capacidad crítica y solo reproducen y aceptan determinados comportamientos o fenómenos sociales por la fuerza que impone la frecuencia, pues los fenómenos que actualmente se presentan de manera frecuente, desde el punto de vista de una ética de la vida, difícilmente pueden ser considerados como un objetivo a ser alcanzado. El análisis de los elementos constitutivos de la normalidad, muestra que los fenómenos que están altamente extendidos dentro de las sociedades actuales, están poniendo en riesgo tanto las democracias, como la reproducción misma de la vida.

La crisis de la normalidad representa: el desmoronamiento de las certezas, que por mucho tiempo han mantenido un sistema socioeconómico que hoy se presenta como insostenible y el cuestionamiento de los valores que han sustentado este hecho. Lo anterior plantea una serie de retos y tareas para la sociedad en su conjunto.

El momento actual lejos de ser un momento apolítico, en donde las sociedades impulsadas por el miedo a la muerte por el contagio al Covid-19, deberían mantenerse en casa, esperando el restablecimiento del orden anterior, de la normalidad anterior, se presenta como un profundo momento político, en donde resulta urgente analizar las características disfuncionales del sistema socioeconómico, para decidir qué es lo realmente deseable para la humanidad, de pensar en la necesidad de una reconstrucción profunda de las sociedades. Para esta reflexión es clave la inacción y el confinamiento, al que sometió la crisis sanitaria, pues ha permitido observar las consecuencias que tiene para la vida en común, los desequilibrios producto de la aceptación de la mercantilización de la misma.

En concordancia con Dussel (2020), se considera que la actual crisis sanitaria pone en contraposición dos principios fundamentales: el principio de la modernidad abstracta, científica y tecnológica y el principio de la vida. Desde este punto de vista, se cuestiona el papel que ha desempeñado el mundo intelectual, al sobrevalorar la racionalidad positivista y al negar otros tipos de conocimientos, que son profundamente humanistas y que tienen mucho que aportar desde la perspectiva de la reconstrucción de una ética y política de la vida.

Al mundo intelectual, se le presenta la tarea de replantearse su rol como sujeto de cambio sociopolítico. Hoy más que nunca, las ciencias antropológicas deberían pensar en volver al humanismo, porque finalmente es el ser humano su objeto de estudio, con todas las contradicciones que eso conlleva. Los intelectuales son responsables de tener coherencia, y de ayudar a crear un nuevo marco conceptual, que permita ver las posibilidades que se presentan en este momento histórico. Entender el papel del lenguaje es crucial para explicar y dar sentido a nuestras realidades. En este sentido, 
todavía es posible observar el uso de dicotomías políticas anacrónicas, que no son apropiadas para al debate actual. El comunismo y el capitalismo se presentan como las dos caras de una moneda, que hacen referencia al sistema de producción como eje central de la vida política, en este sentido cuestionar la producción como fin último de la humanidad, podría considerarse hoy en día como un acto revolucionario pero, sin embargo, podría ser básico para el desarrollo de las sociedades futuras.

Desde el punto de vista de los pueblos, se plantea la tarea de poder dar una respuesta democrática de base. Latinoamérica al igual que el resto de la periferia, es una región gestada en profundas desigualdades, contradicciones y privaciones, que ha sufrido de forma particular el desastre neoliberal, pero también es donde podemos encontrar las respuestas diferentes a este modelo, que permiten repensar el sistema productivo mundial y decidir sobre las tecnologías que se usaran.

La fractura que se puede observar en América Latina, se expresa en los nuevos movimientos sociales. Históricamente ni los gobiernos, ni las elites económicas han concedido derechos, lamentablemente la historia ha enseñado, que es necesario luchar por ellos y en muchas ocasiones sacrificando vidas. La movilización popular es una de las herramientas que se tiene para enfrentar las grandes desigualdades, pero es necesario repensar la protesta social, buscando nuevas estrategias para enfrentar a las fuerzas represoras del estado, que cada vez cuentan con más tecnologías y armamento para hacer frente a un pueblo desarmado. La lógica de la guerra interna es nefasta tanto para las democracias, como para las personas que actúan como escudos humanos en estos enfrentamientos. La normalización de la violencia es peligrosa en muchos sentidos, por lo que es necesario repensar el movimiento popular, renovar el marco conceptual, pensar de forma más novedosa y atractiva sus estrategias y vigencia.

La normalización de la mercantilización de lo público, implica el desplazamiento de los valores centrales y la dificultad de definir los límites entre el sistema económico y el sistema político. Pero hoy, es cada vez más claro, que el mercado no puede, ni debe solucionar los problemas humanos, sino que, lo deben hacer instituciones creadas con fuertes principios éticos que piensen en el bien común y no en la ganancia del capital (Dussel, 2020). Al respecto, es fundamental exigir a las elites políticas, que asuman la responsabilidad que les plantea el momento actual y dejen de lado la discusión sobre las antiguas dicotomías políticas que solo representan a los poderes económicos.

La gestión local de la salud, la importancia de otros tipos de conocimientos, de lo comunitario, de lo presencial, de los lazos de solidaridad que vinculen el territorio con lo cotidiano, son fundamentales para la creación de una nueva normalidad diferente a la de las distopias de la ciencia ficción. La defensa de la vida, se presenta como un presupuesto filosófico y ontológico fundamental, para esto es necesario que la humanidad se perciba como parte de un organismo planetario vivo, con diferentes subsistemas de vida que se afectan mutuamente, ya sea para su supervivencia o extinción.

El análisis que antecede no tiene como objetivo presentar una definición o crítica acabada sobre la normalidad, sino más bien incentivar e inducir, a la profundización de una reflexión que ya se viene gestando hace algún tiempo, pero que hoy gracias a la crisis sanitaria, se presenta como urgente e ineludible, tanto desde las ciencias antropológicas como desde la sociedad en su conjunto.

\section{Referencias Bibliográficas}

Batthyány, K. (2020). Políticas públicas, género y cuidados en América Latina en tiempos de pandemia. Trabajo presentado en el simposio virtual Crisis del Covid-19, transformaciones del Estado y cambio social mundial. 
Canguilhem, G. (1966/1971). Lo normal y lo patológico. Editorial Siglo XXI

Caponi, S. (1998). Lo Normal como categoría sociológica. Trabajo presentado en el $20^{\circ}$ Congreso de Filosofía, Boston.

Cohen, M. (2020). Repensar la ciudad. Trabajo presentado en el simposio virtual Crisis del Covid-19, transformaciones del Estado y cambio social mundial.

Chomsky, N. (2020). Coronavirus ¿Quéestá en juego? Entrevistado por S. Horvat. Mundo después del Coronavirus, DIEM25. Recuperado de: https://www.youtube.com/watch?v=tN3In2rLI4\&feature $=$ emb_logo

Domingues, J. M. (2020). Del riesgo global a la amenaza global: capacidades estatales en tiempos de coronavirus. Trabajo presentado en el simposio virtual Crisis del Covid-19, transformaciones del Estado y cambio social mundial.

Durkheim, E. (1895). Las reglas del método sociológico. Editorial Fondo de Cultura Económica México.

Dussel, E. (2020). Contra la normalidad. Nuevos horizontes políticos en América Latina. Trabajo presentado en el encuentro virtual Ciclo de diálogos: Los días de pandemia.

Hacking, I. (1990/1991). La domesticación del azar. Editorial Gedisa.

Junta de vigilancia mundial de la preparación. (2019). Un mundo en peligro: Informe anual sobre preparación mundial para las emergencias sanitarias. Recuperado de: https://apps. who.int/gpmb/assets/annual_report/GPMB_Annual_Report_Spanish.pdf

Meneses, M. P. (2020). Las injusticias infecciosas, las injusticias epistémicas: pistas para una economía política de la crisis COVID-19 en el sur de África. Trabajo presentado en el simposio virtual Crisis del Covid-19, transformaciones del Estado y cambio social mundial.

Miley. T. (2020). Respuestas democráticas de base a la pandemia del Covid19: una aproximación global. Trabajo presentado en el simposio virtual Crisis del Covid-19, transformaciones del Estado y cambio social mundial.

Tomadoni, C. (2020). Estado, sociedad y territorio en Alemania en la coyuntura del Covid19. Trabajo presentado en el simposio virtual Crisis del Covid-19, transformaciones del Estado y cambio social mundial.

Torres, E. (2020). El futuro del sistema interestatal y de la legitimación política en la sociedad mundial. Trabajo presentado en el simposio virtual Crisis del Covid-19, transformaciones del Estado y cambio social mundial. 